\title{
16 Dermatosen des männlichen Genitales
}

\author{
Frank-Michael Köhn
}

\subsection{Einleitung}

Die Inspektion des männlichen Genitales findet im Rahmen allgemeinärztlicher oder internistischer Untersuchungen nicht immer ausreichende Beachtung. Hinzu kommt, dass Männer nicht selten Veränderungen an ihrem Genitale bemerken, sie dann aber nicht abklären lassen.

Eine andrologische Untersuchung bietet daher die Möglichkeit, relevante Hauterkrankungen des männlichen Genitales zu erkennen. Zudem eröffnet sie dem Mann die Gelegenheit, von sich aus Fragen zu Befunden an Penis oder Skrotum zu stellen. Erkrankungen des Penis können im Rahmen generalisierter Hauterkrankungen auftreten oder lokalisierte Hauterveränderungen sein (English et al. 1997). Differenzialdiagnostisch müssen entzündliche und infektiöse Erkrankungen sowie benigne und maligne Neubildungen unterschieden werden (s. Tab. 1).

Bei andrologischen Untersuchungen auf Erkrankungen des Genitales achten!

\section{Tab. 1 Hautveränderungen des männlichen Genitales}

\section{Infektionen}

papulosquamöse/systemische Dermatosen

Neoplasien

allergische/irritative Kontaktdermatitis

fixe Arzneimittelexantheme

Traumata 


\subsection{Hautveränderungen ohne Krankheitswert}

Physiologische Hautveränderungen am Penis sind gelbliche Talgdrüsen im Bereich der Vorhaut sowie Papillae coronae glandis am Rand der Eichel. Hierbei handelt es sich um teilweise längliche, hautfarben-rötliche Papeln, die mit einer Häufigkeit von bis zu 30\% auftreten können. Die Patienten werden über die Harmlosigkeit des Befundes aufgeklärt; eine Entfernung sollte nur in Ausnahmefällen durch Kryochirurgie oder $\mathrm{CO}_{2}$-Laser erfolgen (Köhn et al. 1999).

Physiologische Veränderungen des Genitales können Patienten massiv verunsichern!

\subsection{Vorhautverengung (Phimose)}

Bei vollständiger Phimose lässt sich die Vorhaut nicht mehr über die Glans penis (Eichel) zurückstreifen, bei unvollständiger Phimose ist das Zurückstreifen der Vorhaut nur unter Schmerzen und mit größerem Kraftaufwand möglich. Folgen sind entzündliche Veränderungen an Glans penis (Balanitis) und Vorhaut (Posthitis) sowie funktionelle Beschwerden mit Schmerzen bei Erektion und Geschlechtsverkehr. Phimosen können bei Neugeborenen durch Verklebungen des inneren Vorhautblattes mit der Eichel noch natürlicherweise auftreten, sollten sich aber innerhalb des ersten Lebensjahres spontan lösen. Manchmal bleiben diese Verwachsungen auch noch bis zum Pubertätsalter erhalten. Davon sind die erworbenen Phimosen abzugrenzen, die sich infolge infektiöser und entzündlicher Erkrankungen der Vorhaut akut (z.B. bei Herpes genitalis) oder chronisch (z.B. bei Lichen sclerosus et atrophicans) entwickeln können.

Die Therapie der Phimose besteht in der Beschneidung (Circumcision). Die Häufigkeit von Beschneidungen bei Neugeborenen ist starken geographischen Schwankungen unterworfen und natürlich auch von religiösen Faktoren abhängig. Circumcisionen reduzieren das Auftreten von Peniskarzinomen und entzündlichen Peniserkrankungen.

\subsection{Infektiöse Hauterkrankungen des männlichen Genitales}

Infektiöse Peniserkrankungen können durch Pilze, Bakterien, Parasiten oder Viren hervorgerufen werden. Mykotische und bakterielle Infektionen werden meistens manifest als Balanitis oder Balanoposthitis. Die häufigsten parasitären Erkrankungen des Penis betreffen solche mit Trichomonaden und Skabies (Krätzmilbe). Nächtlicher Juckreiz und rötliche, teilweise exkoriierte Papeln sind typisch für Skabies.

Eine noch nicht ganz geklärte Stellung nimmt im Zusammenhang infektionsbedingter Peniserkrankungen das Reiter-Syndrom ein, das durch Harnröhrenentzündung (Urethritis), Bindehautentzündung (Konjunktivitis) und Gelenkentzündungen (Arthritis) charakterisiert ist. Bestimmte urogenitale und gastrointestinale Infektionen können es bei genetisch prädisponierten Patienten triggern (HLA-B27 positiv in 90\%). Genitale Infektionen mit Chlamydien sind am häufigsten mit Reiter-Syndrom asso- 
ziiert (50\% der männlichen Patienten). Die Balanitis circinata ist die häufigste Hautveränderung $(12-70 \%)$ bei befallenen Männern. Die Läsionen sind schmerzfrei und erscheinen als gerötete, serpiginöse, manchmal auch erosive randbetonte Plaques. Bei circumcidierten Männern sind die Läsionen trocken und schuppig und ähneln psoriatischen Veränderungen. Auch histopathologisch bestehen Ähnlichkeiten mit der Psoriasis (Köhn et al. 1999; Krause u. Effendy 1998).

\subsubsection{Infektiöse Balanitis und Balanoposthitis}

Infektiöse Entzündungen an Eichel und Vorhaut kommen bei beschnittenen Männern seltener vor als bei Männern mit intakter Vorhaut. Sie sind selten nur durch einen Krankheitserreger ausgelöst. Häufig finden sich in entsprechenden Kulturen neben verschiedenen Bakterien auch Hefepilze (Candida albicans). Begünstigende Faktoren sind ein gleichzeitig bestehender Diabetes mellitus, reduzierte Immunabwehr (z.B. HIV-Infektion, Einnahme von Immunsuppressiva) oder eine Vorhautverengung.

Die Haut von Eichel und innerem Vorhautblatt ist gerötet, manchmal auch erosiv und bei Hefepilzen mit abstreifbaren, weißlichen Belägen bedeckt. In Zusammenhang mit der entzündlichen Reaktion kann es zu Schwellungen von Eichel und Vorhaut mit daraus resultierender Verengung (relative Phimose) kommen.

Die Behandlung besteht bei bakteriellen Infektionen in der Anwendung antiseptischer Lösungen und antibiotischer Cremes. Die Behandlung von Pilzinfektionen besteht in der lokalen Anwendung von antimykotischen Substanzen in Lösungen, antimykotischen Cremes oder auch Pasten. Nur selten (z.B. bei Immunsuppression) wird eine systemische Therapie notwendig.

Differenzialdiagnostisch müssen auch nichtinfektiöse Ursachen für eine Balanitis in Betracht gezogen werden. Hierzu gehören allergische Kontaktreaktionen (z.B. gegenüber Latex in Kondomen) oder auch unspezifische Irritationen durch übertriebene Reinigungsmaßnahmen oder Lokalbehandlungen des Genitales („Overtreatment-Balanitis“).

\subsubsection{Herpes genitalis}

Genitale Herpesinfektionen werden bevorzugt durch Herpesviren Typ II ausgelöst, während der Typ I überwiegend für Infektionen an anderen Hautarealen verantwortlich ist. Herpesviren Typ II werden in der Regel durch Geschlechtsverkehr übertragen. In ca. 95\% gehen dem erstmaligen Auftreten von genitalen Herpesläsionen intime Kontakte mit Partnern voraus, die selbst akute Herpesinfektionen aufgewiesen haben. Die Inkubationszeit beträgt 3-14 Tage. Danach treten gruppiert angeordnete Bläschen auf, deren dünne Blasendecke schnell durch mechanische Faktoren (z.B. Reiben der Unterwäsche) eröffnet wird, sodass mit Krusten belegte Erosionen entstehen können.

Die Viren können in inaktiver Form in den Ganglien sensibler Nerven persistieren und - begünstigt durch Provokationsfaktoren (Allgemeinerkrankungen, Störungen der Immunabwehr) - aktiviert werden. In ca . 50\% tritt der Herpes genitalis wiederholt auf; bei ca. 15\% der Patienten muss mit > 8 Erkrankungsschüben pro Jahr gerechnet werden. 
Bei ausgeprägtem Krankheitsbild sollte oral therapiert werden (s. Tab. 2). Bei häufigen Rezidiven ist eine Langzeitprophylaxe-für mehrere Monate indiziert (s. Tab. 3).

Bei 4 oder mehr Schüben von Herpes genitalis pro Jahr sollte der Patient über die Möglichkeit einer längerfristigen Suppressionsbehandlung aufgeklärt werden.

Tab. 2 Erstbehandlung des Herpes genitalis

\begin{tabular}{|c|c|c|}
\hline & Dosis (täglich) & Therapiedauer \\
\hline Aciclovir & $3 \times 400 \mathrm{mg}$ & 5-10 Tage \\
\hline Aciclovir & $5 \times 200 \mathrm{mg}$ & 5-10 Tage \\
\hline Famciclovir & $3 \times 250 \mathrm{mg}$ & 5-10 Tage \\
\hline Valciclovir & $2 \times 1000 \mathrm{mg}$ & 7-10 Tage \\
\hline Valciclovir & $2 \times 500 \mathrm{mg}$ & 5 Tage \\
\hline
\end{tabular}

Tab. 3 Suppressionsbehandlung des Herpes genitalis bei > 4 Rezidiven/lahr

\begin{tabular}{|c|c|c|}
\hline & Dosis (täglich) & Therapiedauer \\
\hline Aciclovir & $2-3 \times 400 \mathrm{mg}$ & Monate \\
\hline Aciclovir & $4 \times 200 \mathrm{mg}$ & Monate \\
\hline Famciclovir & $2 \times 125-250 \mathrm{mg}$ & Monate \\
\hline Valaciclovir & $1-2 \times 500 \mathrm{mg}$ & Monate \\
\hline
\end{tabular}

\subsubsection{Condylomata acuminata (Feigwarzen)}

Feigwarzen sind verschieden große, rötliche Tumoren mit zerklüfteter Oberfläche (s. Abb. 1). Sie treten beim Mann bevorzugt an der Eichel und Vorhaut (75\%) oder am Penisstamm (25\%) auf, sind aber auch in der Analregion oder an anderen Körperstellen vorzufinden.

Verursacht werden sie durch Humane Papillomviren (HPV). Die Erreger der Feigwarzen sind bevorzugt die Typen 6 und 11; andere HPV-Typen (z.B. 16, 18, 31, 33) wurden

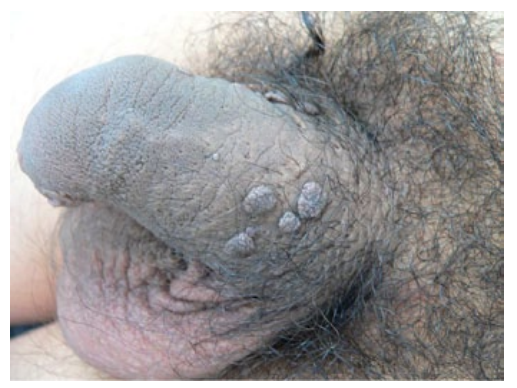

Abb. 1 Condylomata acuminata am Penisstamm 
nicht nur in Condylomata acuminata, sondern auch in bösartigen Neubildungen des Genitaltraktes nachgewiesen. Humane Papillomviren sind deshalb mit der Entstehung von Peniskarzinomen in Zusammenhang gebracht worden. Klinisch manifestieren sich Infektionen mit den HPV Typen 16 und 18 als bowenoide Papulose, flachen, bräunlichen Knötchen, die bereits ein nicht-invasives Karzinom darstellen. Die Therapie besteht in der Entfernung durch Elektrokauterisation, Lasertherapie, Kryotherapie oder durch lokale Anwendung von Podophyllotoxin, Imiquimod oder Extrakt von Grünem Tee. Wichtig sind der Ausschluss anderer sexuell übertragbarer Infektionen (z.B. HIV, Gonorrhoe, Lues, Hepatitis) und die gründliche Untersuchung der Sexualpartner.

\subsection{4 „Klassische“ Geschlechtskrankheiten}

Unter den klassischen Geschlechtskrankheiten wurden früher Syphilis, Gonorrhoe (Tripper), Ulcus molle (weicher Schanker) und Lymphogranuloma inguinale verstanden (Köhn et al. 1999). Die beiden zuletzt genannten Erkrankungen finden sich in Deutschland nur sehr selten. Mit wesentlich häufigerem Auftreten ist in tropischen Regionen oder großen europäischen Hafenstädten zu rechnen. Der weiche Schanker ist eine bakterielle Infektion mit Haemophilus Ducreyi; 2-5 Tage nach der Infektion bildet sich am Penis ein schmerzhaftes Geschwür mit Schwellung der Leistenlymphknoten.

\section{Gonorrhoe}

Die Gonorrhoe des Mannes ist eine bakterielle Infektion durch Neisseria gonorrhoeae, die vorzugsweise die Harnröhre befällt (unkomplizierte Gonorrhoe). In Abhängigkeit zu den sexuellen Praktiken können Proktitis, Pharyngitis oder Konjunktivitis auftreten.

Nach einer Inkubationszeit von 2-10 Tagen kommt es vor allem am Morgen vor der ersten Entleerung von Urin zu eitrigem Ausfluss aus der Harnröhre („Bonjourtropfen“). Dadurch können Eichel und Vorhaut gereizt werden, sodass sich eine Balanitis oder Balanoposthitis entwickeln. Die entzündlichen Veränderungen begünstigen ebenso zusätzliche Infektionen mit humanen Papillomviren. Als Folge einer komplizierten Gonorrhoe führen aufsteigende Infektionen beim Mann zu Prostatitis und Epididymitis. Die seltene hämatogene Streuung geht mit Arthritis, Perihepatitis gonorrhoica, Hautveränderungen, Endokarditis oder Meningitis einher.

Eine frühzeitige antibiotische Therapie führt zu sicherer und folgenloser Abheilung der Gonorrhoe. Resistenzentwicklungen der Erreger gegen die Antibiotika müssen aber in Betracht gezogen werden.

\section{Lues (Syphilis)}

Erreger der Lues ist ein spiralig gewundenes Bakterium (Treponema pallidum), das nach einer Inkubationszeit von 3-4 Wochen am Ort des Erregereintritts zu einem „Primäraffekt“ mit Schwellung der entsprechenden Lymphknoten führt. In Abhängigkeit von der ausgeübten Sexualpraktik können solche „Primäraffekte“ überall am Körper auftreten. Aus einem anfänglichen Knötchen entsteht ein scharf begrenztes, gelblich belegtes, schmerzloses, hochinfektiöses Geschwür. Da der Rand hart ist, 
wird die Erkrankung auch als „harter Schanker“ vom „weichen Schanker“ abgegrenzt. Idealerweise wird die Syphilis noch in diesem ersten Stadium erkannt und antibiotisch behandelt. Erfolgt keine suffiziente Therapie, entwickelt sich nach 2-6 Monaten eine sekundäre Syphilis (Lues II), die mit Veränderungen der Haut (Exantheme, Leukoderme), Lymphknoten (Lymphadenopathie), Schleimhäute (Plaques muqueuses der Zunge, anogenitale Condylomata lata) und der Haare (Alopzie) einhergeht. Die Veränderungen heilen klinisch auch ohne Therapie ab. Unbehandelt schreitet die Syphilis aber nach 3-5 Jahren in das dritte Stadium fort (Lues III). Dieses manifestiert sich in 30-40\% mit granulomatösen Veränderungen der Haut und Gefäße mit z.T. ernsten Komplikationen. Nach weiteren Jahren ohne suffiziente Therapie folgt schließlich die quartäre Syphilis mit neurologischen Symptomen und Persönlichkeitsveränderungen.

\subsection{Lichen sclerosus et atrophicus}

Der Lichen sclerosus et atrophicus ist eine chronisch-entzündliche Bindegewebserkrankung der Haut, die bevorzugt die Anogenitalregion befällt. Die Ursache der Erkrankung ist unklar; sie kann mit einem Diabetes mellitus assoziiert sein. Zusätzlich werden traumatische Faktoren, Autoimmunerkrankungen, genetische Faktoren sowie hormonelle Faktoren diskutiert. Betroffen sind meist unbeschnittene Patienten im mittleren Lebensalter (Köhn et al. 1999; Krause u. Effendy 1998).

Klinisch präsentiert sich die Erkrankung mit porzellanartig verfärbten, derben, zunächst kleinfleckigen, dann konfluierenden Herden, die schließlich zu größeren Flächen mit pergamentartiger Oberfläche konfluieren können. Bei längerem Bestehen werden die Patienten durch z.T. quälenden Juckreiz beeinträchtigt. Im weiteren Verlauf der Erkrankung kommt es beim Mann zu einer narbigen Verengung der Vorhaut und sekundären entzündlichen Veränderungen (s. Abb. 2). Bei chronischem Verlauf des Lichen sclerosus et atrophicans kann in den Herden ein spinozelluläres Karzinom auftreten. Therapeutisch können die Beschneidung und die Anwendung steroidhaltiger Cremes sowie eine Abtragung einzelner Herde mit dem $\mathrm{CO}_{2}$-Laser erwogen werden. Auch lokale Anwendung von Tacrolimus oder Pimecrolimus kann zu Besserung führen.

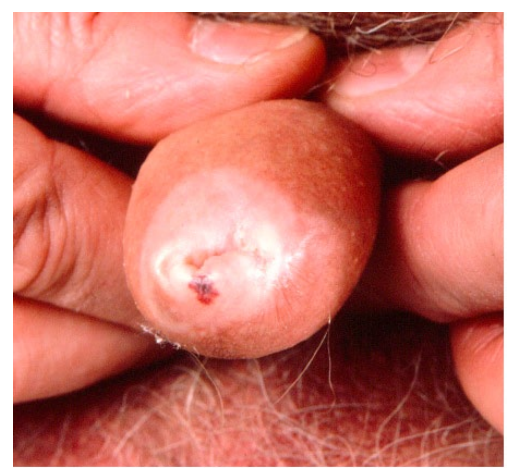

Abb. 2 Lichen sclerosus et atrophicus mit absoluter Phimose 


\subsection{Balanitis circumscripta plasmacellularis (Morbus Zoon)}

Die Balanitis circumscripta plasmacellularis ist eine chronische Erkrankung, die bevorzugt unbeschnittene Männer im mittleren oder höheren Alter befällt. Mangelnde Hygiene, chronische bakterielle Infektionen, immunologische Faktoren sowie physikalische Faktoren wie Hitze oder Traumatisierungen sind als Ursachen diskutiert worden. Die Plasmazellbalanitis beginnt mit solitären, glänzenden, rötlichen Herden an Glans penis oder Vorhaut. Die Behandlung kann mit Kortikosteroiden, Circumcision, antimikrobiellen Substanzen oder $\mathrm{CO}_{2}$-Laser erfolgen.

\subsection{Generalisierte Hautveränderungen mit Manifestationen am männlichen Genitale}

Eine Vielzahl auch die übrige Haut befallender Hauterkrankungen betrifft ebenfalls den Penis. Hierzu gehören Veränderungen z.B. bei Schuppenflechte, Vitiligo, blasenbildenden Erkrankungen, Lichen ruber oder Urticaria.

\subsection{Fixe Arzneimittelexantheme}

Nach Sensibilisierung gegenüber einem Medikament manifestieren sich fixe Arzneimittelreaktionen als solitäre oder multiple, scharf begrenzte erythematöse Maculae oder Plaques, in denen sich auch Blasen entwickeln können. Seltener werden Ulzerationen beobachtet. Typischerweise treten diese Veränderungen nach Einnahme des gleichen Medikamentes immer wieder an derselben Stelle auf und heilen innerhalb von 2-3 Wochen mit postinflammatorischer Hyperpigmentierung ab. Substanzen, die fixe Arzneimittelexantheme verursachen können, sind: Tetrazykline, Doxycyclin, Penicilline, Phenolphthalein, Sulfonamide, Barbiturate, Salizylate, Dapson, Griseofulvin, Carbamazepin, Dimenhydrinat, Metamizol, Hydroxyzinhydrochlorid und Colchicin.

\subsection{Maligne Veränderungen des Penis}

Neubildungen am Penis können harmloser Natur sein (z.B. Fibrome, Hämangiome, Pigmentflecken); differenzialdiagnostisch müssen aber auch bösartige Neubildungen berücksichtigt werden (Köhn et al. 1999; Krause u. Effendy 1998). Dabei können diese Veränderungen primär am Penis auftreten (z.B. Melanome, spinozelluläre Karzinome) oder sich erst sekundär im Rahmen eines allgemeinen Hautbefalls am Penis manifestieren (z.B. kutane Lymphome). Andere Hauterkrankungen des Penis können den malignen Neoplasien vorausgehen (z.B. Lichen sclerosus et atrophicans).

\subsubsection{Erythroplasie Queyrat}

Die Erythroplasie Queyrat ist ein nicht-invasives Karzinom. Klinisch fallen bei älteren, nicht beschnittenen Männern scharf begrenzte, unregelmäßig konfigurierte 
Rötungen auf, die nass-glänzend oder fein-granulierend erscheinen. Die Hautveränderungen sollten chirurgisch oder durch Lasertherapie entfernt werden.

\subsubsection{Invasives Plattenepithelkarzinom des Penis („Peniskarzinom“)}

Plattenepithelkarzinome des Penis sind selten. Sie haben nur einen Anteil von o,3$0,5 \%$ an allen Karzinomen des Mannes. Häufig bestehen prädisponierende Faktoren wie Infektionen mit humanen Papillomviren (HPV 16 und 18), chronische Entzündungen oder Phimosen. Im Bereich der Eichel oder Vorhaut kommt es zum Wachstum eines rötlichen Tumors mit Blutungsneigung (s. Abb. 3). Später kann es zur Metastasenbildung kommen. Die operative Entfernung des Karzinoms sollte deshalb frühzeitig erfolgen.

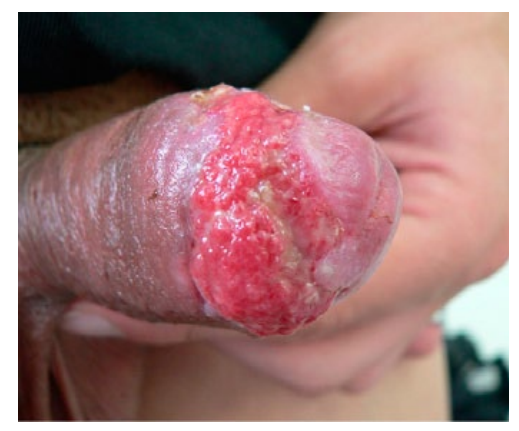

Abb. 3 Peniskarzinom

\section{Literatur}

English JC, Laws RA, Keough GC, Wilde JL, Foley IP, Elston DM (1997) Dermatoses of the glans penis and prepuce. I Am Acad Dermatol 37, 1-24

Köhn FM, Pflieger-Bruss S, Schill WB (1999) Penile skin diseases. Andrologia 31, 3-11

Krause W, Effendy I (1998) Genitale Hautkrankheiten. Ferdinand Enke Verlag, Stuttgart 